Supporting Information:

\title{
Expedient Construction of the Ziegler Intermediate Useful for the Synthesis of Forskolin via Consecutive Rearrangements
}

\author{
Heping Ye, Gang Deng, Jun Liu and Fayang G. Qiu*
}

Guangzhou Institute of Biomedicine and Health, The Chinese Academy of SciencesGuangzhou, 510663,

China; and Department of Chemistry, Wuhan University of Science and Technology, Wuhan, 430070,

$$
\text { China }
$$

qiu_fayang@gibh.ac.cn

Table of Contents

General Information--_-

Experimental Procedures and characterization data--_o-_-_-_-S6

NMR spectra- 


\section{General Information:}

All ${ }^{1} \mathrm{H} \&{ }^{13} \mathrm{C}$ NMR spectra were recorded on Bruker AV-400 (400 MHz) instrument. Mass spectra were obtained on an API3000 LCMS with electrospray ionization. General chemicals were obtained from commercial suppliers and were used without further purification. Triethylamine and dichloromethane were dried with active $4 \AA$ molecular sieves. Acetonitrile was distilled over $\mathrm{CaH}_{2}$ and methanol was dried using magnesium turnings.

\section{Experimental Procedures and characterization data:}

(E)-4-(2, 6, 6-trimethyl-2,3-epoxycyclohexyl)-3-buten-2-one ${ }^{1}$<smiles>CC(=O)/C=C/C1C(C)(C)CCC2OC21C</smiles>

A solution of $\alpha$-ionone $57.6 \mathrm{~g}(0.3 \mathrm{~mol})$ in dichloromethane $(300 \mathrm{~mL})$ was cooled to $5{ }^{\circ} \mathrm{C}$, 3-chloroperbenzoic acid $(72.5 \mathrm{~g}, 75 \%, 0.315 \mathrm{~mol})$ was slowly added. The mixture was filtered after being stirred for 1 hour below $10^{\circ} \mathrm{C}$. The filtrate was successively washed with aqueous sodium sulfite $(10 \%$, $100 \mathrm{~mL})$, sodium hydroxide $(5 \%, 100 \mathrm{~mL})$, water $(100 \mathrm{~mL})$ and brine $(50 \mathrm{~mL})$. After being dried over $\mathrm{MgSO}_{4}$ and filtration, the solvent was removed under reduced pressure and the epoxide (59 $\mathrm{g}, 95 \%$ ) was obtained as a clear, colorless oil. The product was used for the next step without further purification.

\section{(3E)-4-(2, 6, 6-trimethyl-3-hydroxy-1-cyclohexen-1-yl)-3-buten-2-one (5) ${ }^{1}$}<smiles>CC(=O)/C=C/C1=C(C)C(O)CCC1(C)C</smiles>

A solution of the epoxide obtained as described above $(59 \mathrm{~g}, 0.28 \mathrm{~mol})$ in methanol $(250 \mathrm{~mL})$ was treated with sodium methoxide (prepared from $\mathrm{Na}, 1.74 \mathrm{~g}$ and $\mathrm{MeOH}, 15 \mathrm{~mL}$ ), and the mixture was stirred at reflux for $4 \mathrm{~h}$. The mixture was then cooled to room temperature, treated with acetic acid $(\sim 3 \mathrm{~mL})$ until

(1) Rosenberger, M.; McDougal, P.; Bahr, J. J. Org. Chem. 1982, 47, 2130 
$\mathrm{pH}=7$. Methanol was removed under reduced pressure and water $(300 \mathrm{~mL})$ was added to the residue. The mixture was extracted with ethyl acetate $(3 \times 100 \mathrm{~mL})$ and the organic layers were combined and washed with brine $(50 \mathrm{~mL})$, dried over $\mathrm{MgSO}_{4}$. After filtration, the solvent was removed under reduced pressure. The residue was subjected to flash chromatography on silica gel, eluting with a mixture of ethyl acetate / petroleum ether (1:4), to give product 5 (48 g, 81\%) as a red oil.

\section{3-Oxo-butyric acid -2, 4, 4-trimethyl-3-((E)-3-oxo-but-1-enyl)-cyclohex-2-enyl ester ${ }^{2}$}<smiles>CC(=O)/C=C/C1=C(C)C(OC(C)=O)CCC1(C)C</smiles>

To a solution of $5(6.03 \mathrm{~g}, 0.029 \mathrm{~mol})$ in dichloromethane $(600 \mathrm{~mL})$ was added triethylamine $(5.86 \mathrm{~g}$, $0.058 \mathrm{~mol})$. The mixture was cooled to $0{ }^{\circ} \mathrm{C}$ and diketene $(4.88 \mathrm{~g}, 0.058 \mathrm{~mol})$ was slowly added. After the mixture was stirred at $0{ }^{\circ} \mathrm{C}$ for 3 hours, water $(200 \mathrm{~mL})$ was added. After separation, the aqueous phase was extracted with dichloromethane $(2 \times 50 \mathrm{~mL}) . \quad$ The organic phases were combined and washed with brine (20 mL), and dried over $\mathrm{MgSO}_{4}$. The solvent was removed under reduced pressure. Purification by using flash chromatography on silica gel, eluting with a mixture of ethyl acetate / petroleum ether $(1: 5 \mathrm{v} / \mathrm{v})$ gave the desired product $(7.63 \mathrm{~g}, 90 \%)$ as a yellow crystalline solid.

\section{3,6,6,8b-Tetramethyl-2a,6,7,8,8a,8b-hexahydro-2H-naphtho[1,8-bc]furan-2-one(6) ${ }^{2}$}<smiles>CC1=CC=C2[C@@H](C)CC[C@@H]3OC(=O)[C@@H]1C23C</smiles>

To a mixture of $\mathrm{Cs}_{2} \mathrm{CO}_{3}(0.65 \mathrm{~g}, 2 \mathrm{mmol})$ and $\mathrm{K}_{2} \mathrm{CO}_{3}(1.12 \mathrm{~g}, 8 \mathrm{mmol})$ were added a solution of the product from the previous step $(2 \mathrm{~g}, 8 \mathrm{mmol})$ in $500 \mathrm{~mL}$ of dry $\mathrm{CH}_{3} \mathrm{CN}$. The mixture was refluxed for 12 h. $\mathrm{CH}_{3} \mathrm{CN}$ was removed and water $(60 \mathrm{~mL})$ was added. The mixture was extracted with ethyl acetate $(3 \times 15 \mathrm{~mL})$. The combined organic phase was washed with brine $(10 \mathrm{~mL})$, and dried over $\mathrm{MgSO}_{4}$. The solvent was removed under reduced pressure. Purification using flash chromatography on silica gel, eluting with a mixture of ethyl acetate / petroleum ether (1:10), provided product 6 (763 $\mathrm{mg}, 48 \%)$ as a 
white crystalline solid.

\section{3,4-Epoxy-3,6,6,8b-tetramethy-2a,6,7,8,8a,8b-hexahydro-2H-naphtho[1,8-bc]furan-2-one $(4)^{3}$}

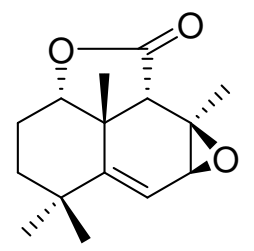

To a solution of lactone 6 (930 mg, $4.0 \mathrm{mmol})$ in dichloromethane $(30 \mathrm{~mL})$ was slowly added 3-chloroperoxybenzoic acid $(966 \mathrm{mg}, 4.2 \mathrm{mmol})$ at $0{ }^{\circ} \mathrm{C}$, and the mixture was stirred for 12 hours at $0{ }^{\circ} \mathrm{C}$. Potassium fluoride $(618 \mathrm{mg}, 8.3 \mathrm{mmol}$ ) was added and the mixture was filtered. The filtrate was washed with saturated aqueous sodium bicarbonate $(2 \times 10 \mathrm{~mL})$ and then brine $(10 \mathrm{~mL})$. The organic phase was dried over $\mathrm{MgSO}_{4}$. The solvent was removed under reduced pressure. The residue was purified by using flash chromatography on silica gel, eluting with a mixture of ethyl acetate / petroleum ether (1:8) to afford epoxide 4 (795 mg, 80\%) as white crystalline solid.

${ }^{1}$ H NMR: $\delta\left(400 \mathrm{MHz}, \mathrm{CDCl}_{3}\right.$, ppm): 5.94 (d, H-6, $\left.J=4.8 \mathrm{~Hz}, 1 \mathrm{H}\right), 4.20(\mathrm{t}, \mathrm{H}-1, J=2.8 \mathrm{~Hz}, 1 \mathrm{H}), 3.26$ (d, H-7 , J = 4.8 Hz, $1 \mathrm{H}), 2.75$ (s, H-9, $1 \mathrm{H}), 1.71(\mathrm{~s}, 3 \mathrm{H}), 1.46(\mathrm{~s}, 3 \mathrm{H}), 1.12(\mathrm{~s}, 3 \mathrm{H}), 1.11(\mathrm{~s}, 3 \mathrm{H})$.

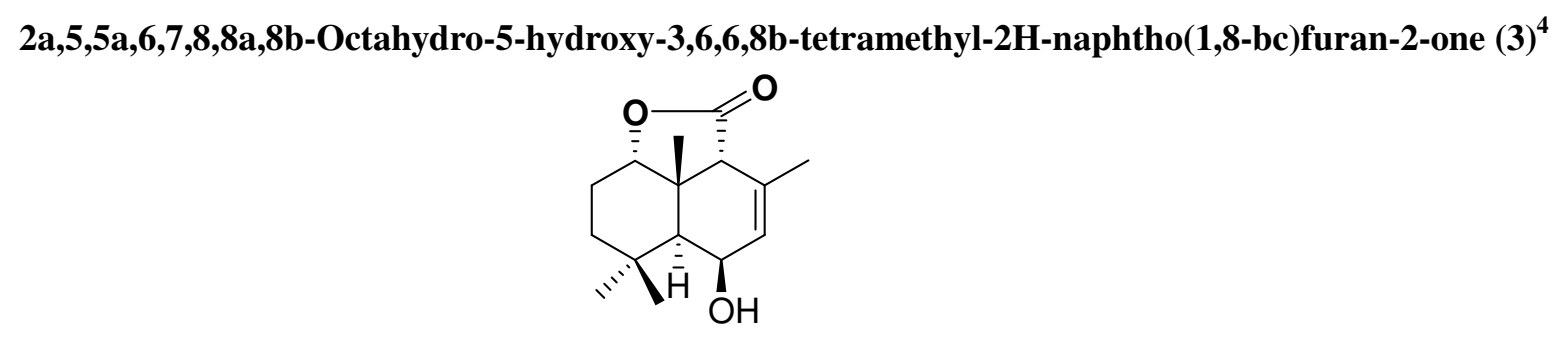

A solution of $4(2 \mathrm{~g}, 8 \mathrm{mmol})$, TsNHNH $2(5.95 \mathrm{~g}, 32 \mathrm{mmol})$ and TsOH $(137.6 \mathrm{mg}, 0.8 \mathrm{mmol})$ in THF(30 $\mathrm{mL}$ ) was stirred at reflux until the reaction reached completion $(2 \mathrm{~h})$ as determined by the absence of the starting material on TLC. Ether $(200 \mathrm{~mL})$ and then aqueous hydrochloric acid $(1 \mathrm{M}, 32 \mathrm{~mL})$ was added. The aqueous phase was extracted with ether $(3 \times 10 \mathrm{~mL})$, the combined organic phase was washed respectively with saturated sodium bicarbonate $(30 \mathrm{~mL})$, water $(3 \times 20 \mathrm{~mL})$, and brine $(20 \mathrm{~mL})$ and dried over $\mathrm{MgSO}_{4}$. After filtration, the solvent was removed at room temperature on a rotary evaporator to provide a white powder, which was treated with sulfuric acid in the following operation. However, pure 3 ' may be obtained by using flash chromatography on silica gel, eluting with a mixture of ethyl acetate / petroleum ether $(1: 5 \mathrm{v} / \mathrm{v})$.

${ }^{1}$ H NMR: $\delta\left(400 \mathrm{MHz}, \mathrm{CDCl}_{3}, \mathrm{ppm}\right): 5.82(\mathrm{dd}, \mathrm{H}-7, J=8.0 \mathrm{~Hz}, J=4.0 \mathrm{~Hz}, 1 \mathrm{H}), 5.66(\mathrm{dd}, \mathrm{H}-6, J=8.0 \mathrm{~Hz}$, $J=4.0 \mathrm{~Hz}, 1 \mathrm{H}), 4.18(\mathrm{t}, \mathrm{H}-\mathrm{l}, 1 \mathrm{H}), 2.60(\mathrm{~s}, \mathrm{H}-9,1 \mathrm{H}), 1.60(\mathrm{~s}, 3 \mathrm{H}), 1.26(\mathrm{~s}, 3 \mathrm{H}), 0.95(\mathrm{~s}, 3 \mathrm{H}), 0.93(\mathrm{~s}, 3 \mathrm{H})$ 
${ }^{13}$ C NMR: $\delta\left(100 \mathrm{MHz}, \mathrm{CDCl}_{3}, \mathrm{ppm}\right): 174.9,134.8,125.6,80.8,69.1,62.6,42.9,42.5,34.6,31.2,31.2$, $27.0,21.2,20.9,18.8$.

MS(ESI): $[\mathbf{M}+\mathbf{H}]^{+} 251.1$

The above residue was dissolved in dioxane $(200 \mathrm{~mL})$ and stirred with aqueous $0.7 \%$ sulfuric acid $(200 \mathrm{~mL})$ at room temperature for $5 \mathrm{~h}$. The mixture was extracted with ether $(4 \times 100 \mathrm{~mL})$. The ethereal extracts were combined, and consecutively washed with saturated sodium bicarbonate $(80 \mathrm{~mL})$, water $(3 \times 20 \mathrm{~mL})$, and then brine $(20 \mathrm{~mL})$. The organic phase was dried over $\mathrm{MgSO}_{4}$. The solvent was removed at room temperature on a rotary evaporator. Purification by using flash chromatography on silica gel, eluting with a mixture of ethyl acetate / petroleum ether (1:8) gave $\mathbf{3}(1 \mathrm{~g}, 50 \%)$ as a white crystalline solid.

${ }^{1}$ H NMR: $\delta\left(400 \mathrm{MHz}, \mathrm{CDCl}_{3}, \mathrm{ppm}\right): 5.73$ (d, H-7, $\left.J=4.8 \mathrm{~Hz}, 1 \mathrm{H}\right), 4.44(\mathrm{~d}, \mathrm{H}-6, J=3.6 \mathrm{~Hz}, 1 \mathrm{H}), 4.18$ (s, H-1, 1 H) 2.65 (s, H-9, 1 H), 1.96 (s, 3 H), 1.41 (s, 3 H), 1.24 (s, 3 H), 1.05 (s, 3 H).

${ }^{13}$ C NMR: $\delta\left(100 \mathrm{MHz}, \mathrm{CDCl}_{3}, \mathrm{ppm}\right): 176.2,130.2,127.1,84.2,64.7,58.8,45.7,40.8,37.1,32.5,31.1$, $22.7,22.2,21.4,19.4$.

MS(ESI): $[\mathbf{M}+\mathbf{H}]^{+} 251.1$

2a,5,5a,6,7,8,8a,8b-Octahydro-5-hydroxy-3,6,6,8b-tetramethyl-2H-naphtha[1,8-bc]furan-2-one $(7)^{4}$

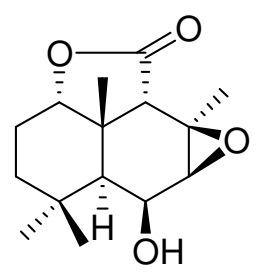

To a solution of $\mathbf{3}(0.2 \mathrm{~g}, 0.8 \mathrm{mmol})$ in dichloromethane $(6 \mathrm{~mL})$ was slowly added 3-chloroperbenzoic acid $(207 \mathrm{mg}, 1.2 \mathrm{mmol})$ at $0{ }^{\circ} \mathrm{C}$. The mixture was allowed to warm to room temperature and was stirred for 72 hours. Potassium fluoride $(138 \mathrm{mg}, 2.37 \mathrm{mmol})$ was added and the mixture was filtered. The filtrate was washed with saturated sodium bicarbonate $(2 \times 2 \mathrm{~mL})$ followed by brine $(1 \mathrm{~mL})$. The organic phase was dried over $\mathrm{MgSO}_{4}$. The solvent was removed under reduced pressure. The residue was purified by using flash chromatography on silica gel, eluting with a mixture of ethyl acetate / petroleum ether (1:5), to furnish epoxide 7 (126 mg, 59\%) as white crystalline solid.

${ }^{1}$ H NMR: $\delta\left(400 \mathrm{MHz}, \mathrm{CDCl}_{3}, \mathrm{ppm}\right): 4.55$ (m, H-6, $\left.1 \mathrm{H}\right), 3.99$ (s, H-1, $\left.1 \mathrm{H}\right), 3.37$ (d, H-7, J = 5.2 Hz, $\left.1 \mathrm{H}\right)$, 2.75 (s, H-9, 1 H), 1.67 (s, $3 \mathrm{H}), 1.45$ (s, $3 \mathrm{H}), 1.21$ (s, $3 \mathrm{H}), 1.08$ (s, $3 \mathrm{H})$. 
4,5-Dihydroxy-3,6,6,8b-tetramethyl-4,5,5a,6,7,8,8a,8b-octahydro-naphtho[1,8-bc]furan-2-one ${ }^{4}$

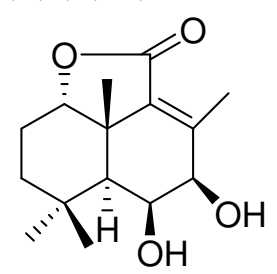

A solution of 7 (119.5 mg, $0.453 \mathrm{mmol}), \mathrm{KOH}(101.4 \mathrm{mg}, 1.811 \mathrm{mmol})$ in methanol (4 mL) was stirred for $10 \mathrm{~min}$ at room temperature, and then treated with hydrochloric acid $(1 \mathrm{M}, 1 \mathrm{~mL})$ until $\mathrm{pH}=7$. Methanol was removed under reduced pressure, and water $(20 \mathrm{~mL})$ was added. The solution was extracted with ethyl acetate $(3 \times 5 \mathrm{~mL})$. The organic phases were combined and washed with brine $(2 \mathrm{~mL})$, before being dried over $\mathrm{MgSO}_{4}$. After filtration, the solvent was removed under reduced pressure. Purification by using flash chromatography on silica gel, eluting with a mixture of ethyl acetate / petroleum ether (1:2), provided product 8 ( $83.7 \mathrm{mg}, 70 \%)$ as white crystalline solid.

${ }^{1}$ H NMR: $\delta\left(400 \mathrm{MHz}, \mathrm{CDCl}_{3}, \mathrm{ppm}\right): 4.44$ (m, H-6, $\left.1 \mathrm{H}\right), 4.16$ (m, H-7, $\left.1 \mathrm{H}\right), 4.06$ (m, H-1, $\left.1 \mathrm{H}\right), 2.21$ (s, 3 H), $1.50(\mathrm{~s}, 3 \mathrm{H}), 1.23(\mathrm{~s}, 3 \mathrm{H}), 1.01(\mathrm{~s}, 3 \mathrm{H})$.

\section{1,16,8,8,9c-Hexamethyl-2,3,3a,5,6a,9a,9b,9c-Octahydro-1H-4,7,9-trioxa-cyclopenta[c]acenaphthylen-} 5-one (2) ${ }^{4}$

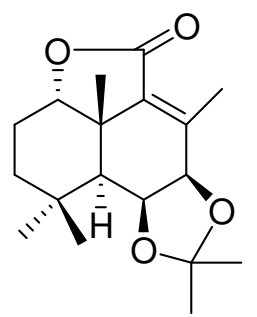

A solution of $8(83.7 \mathrm{mg}, 0.32 \mathrm{mmol})$, TsOH (2.8 mg, $0.016 \mathrm{mmol})$ in 2,2-dimethoxypropane ( $3 \mathrm{~mL})$ was stirred for $90 \mathrm{~min}$ at room temperature. Water $(20 \mathrm{~mL})$ was added, and the mixture was extracted with ethyl acetate $(3 \times 5 \mathrm{~mL})$. The combined organic phase was washed with brine $(2 \mathrm{~mL})$, and then dried over $\mathrm{MgSO}_{4}$. After filtration, the solvent was removed under reduced pressure and a white solid $2(96 \mathrm{mg}$, 99\%) was obtained.

${ }^{1}$ H NMR: $\delta\left(400 \mathrm{MHz}, \mathrm{CDCl}_{3}\right.$, ppm): 4.61 (dd, H-6, J= 7.2 Hz, J = 3.2 Hz, $\left.1 \mathrm{H}\right), 4.57$ (d, H-7, J = 7.2 Hz, $1 \mathrm{H}), 4.07$ (dd, H-l, $J=11.2 \mathrm{~Hz}, J=5.6 \mathrm{~Hz}, 1 \mathrm{H}), 2.27$ (s, $3 \mathrm{H}), 1.93$ (m, $2 \mathrm{H}), 1.60$ (br d, $1 \mathrm{H}), 1.50$ (s, 3 H), 1.41 (s, $3 \mathrm{H}), 1.38$ (s, $3 \mathrm{H}), 1.32(\mathrm{~m}, 2 \mathrm{H}), 1.19$ (s, $3 \mathrm{H}), 1.06$ (s, $3 \mathrm{H})$.

${ }^{13}$ C NMR: $\delta\left(100 \mathrm{MHz}, \mathrm{CDCl}_{3}, \mathrm{ppm}\right): 169.1,144.5,136.5,109.1,87.3,79.1,73.0,51.4,40.3,36.0,32.1$, $31.028 .2,26.1,26.1,25.0,22.7,17.7$. 
MS(ESI):[M+H] ${ }^{+}: 307.2$

NMR spectra: 


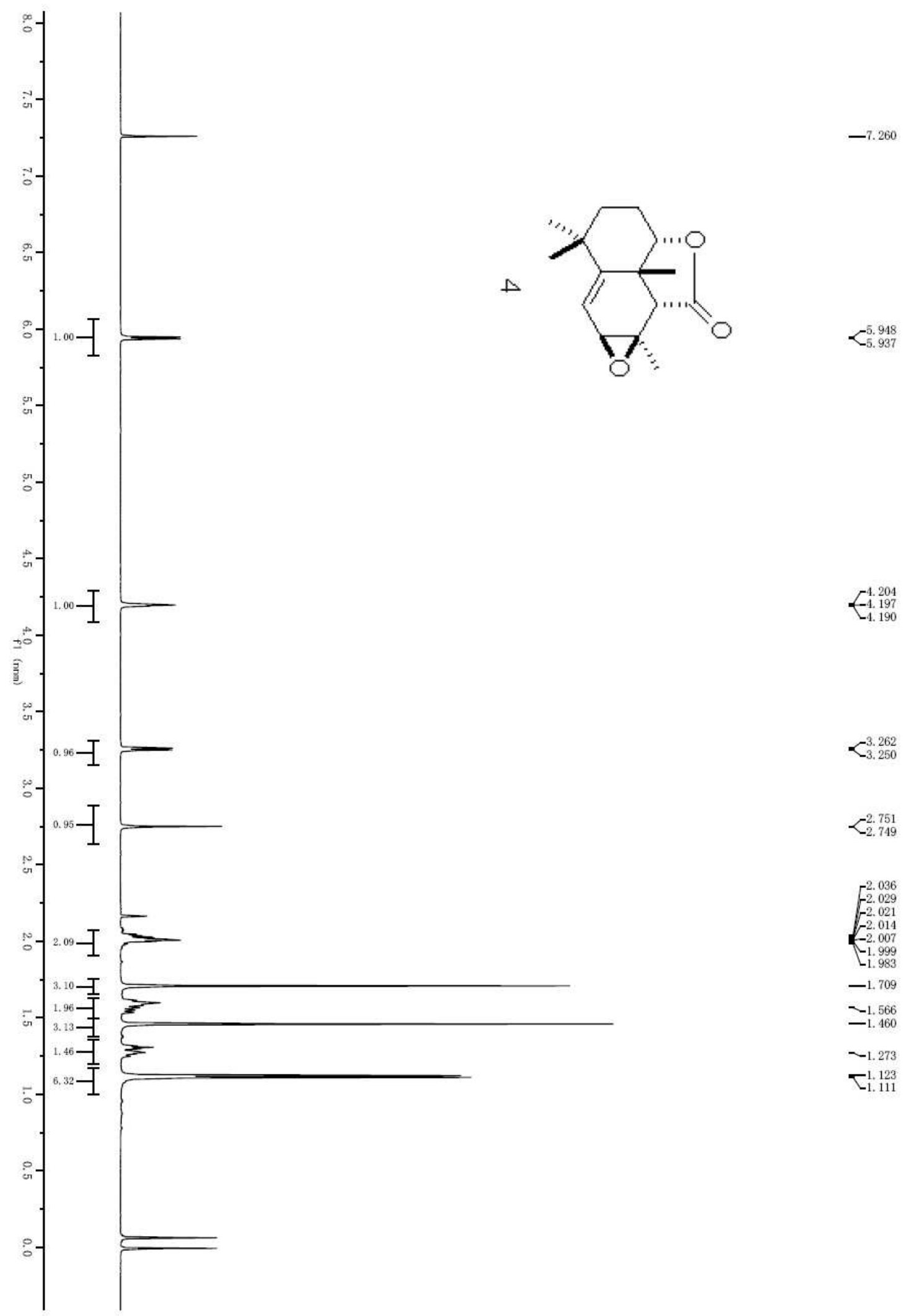




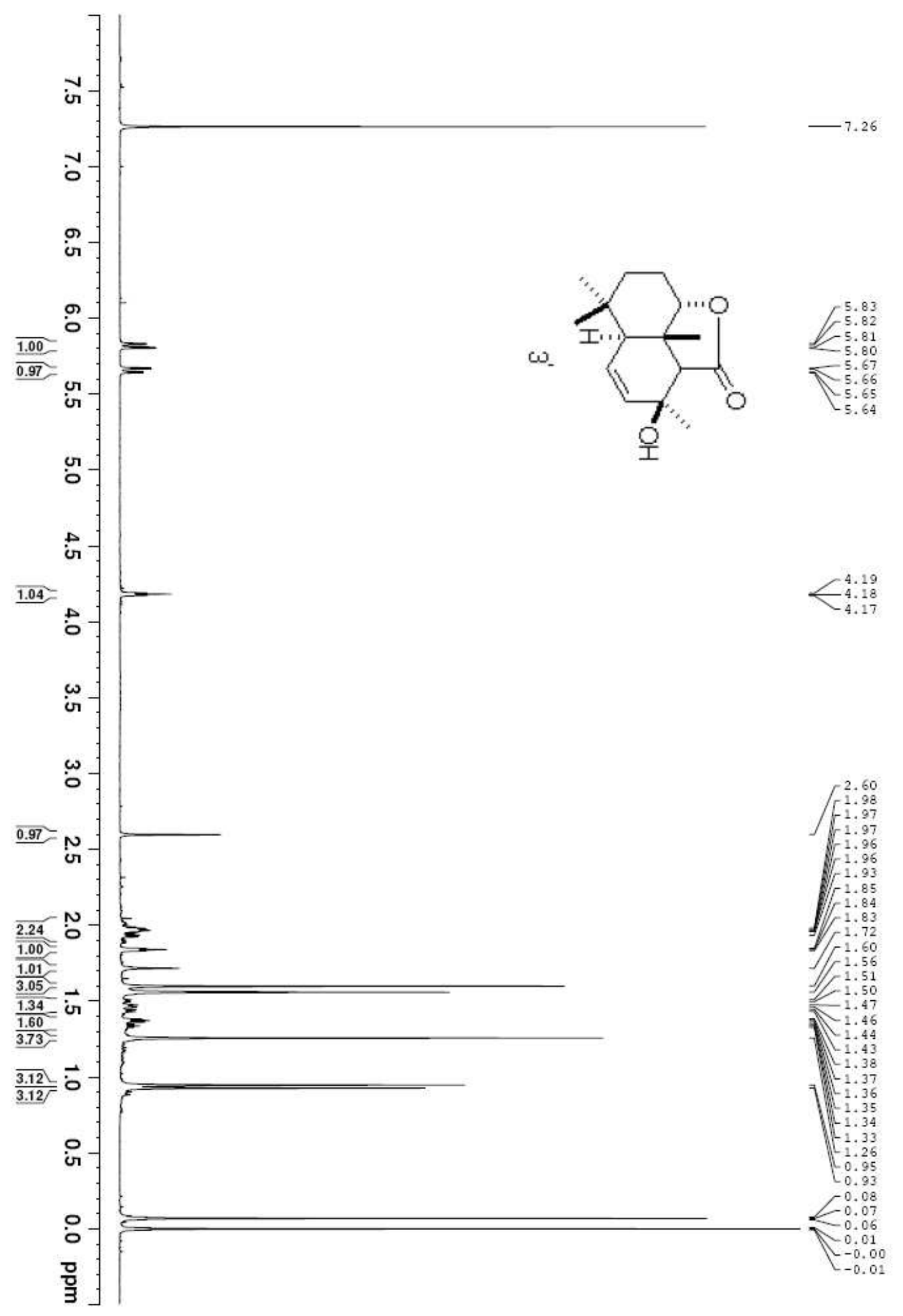



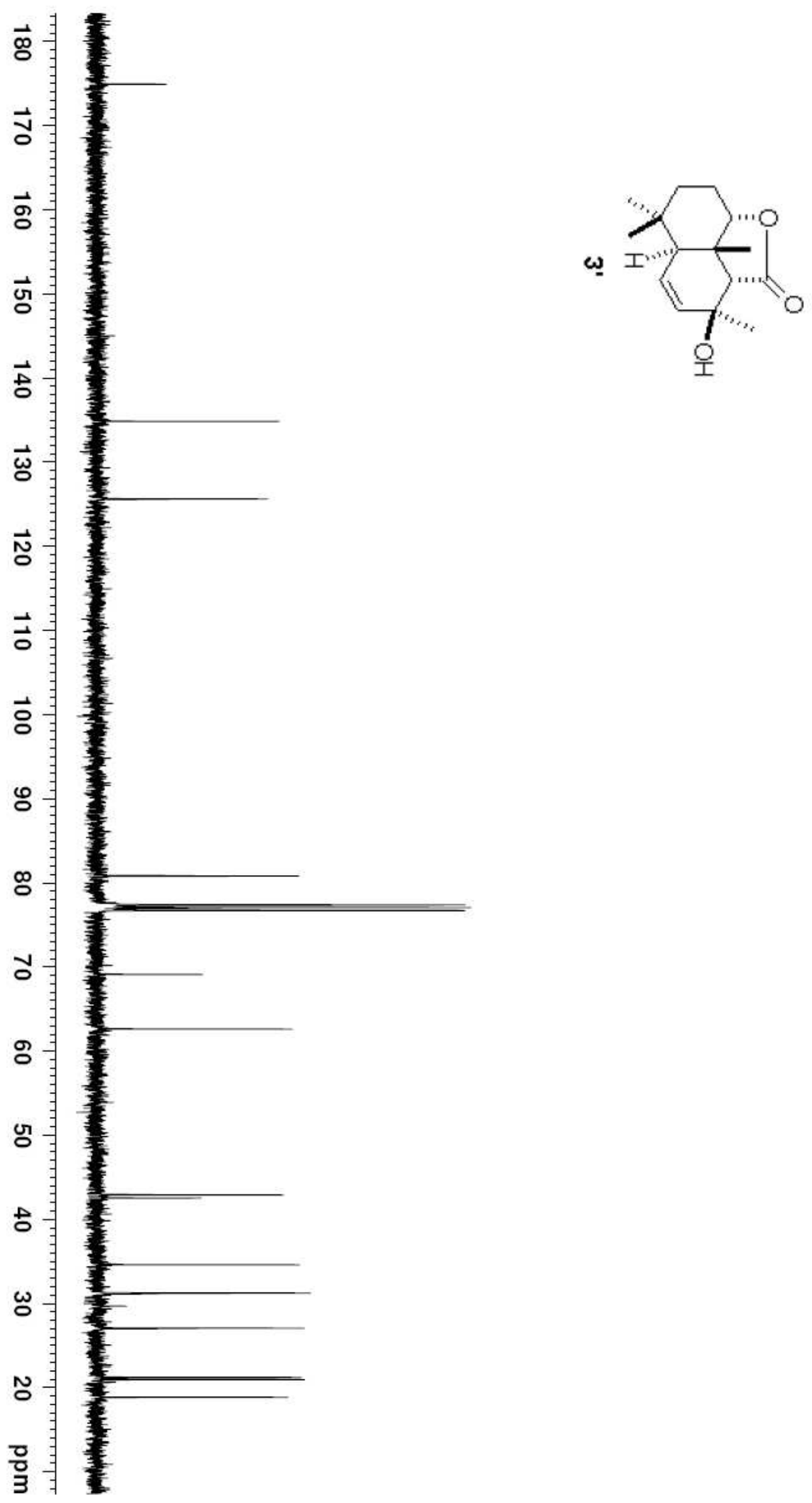

$-134.85$

$-125.61$

$-80.81$

$-77.36$

-77.04
-76.73

$-69.08$

$-62.61$

-42.89
-42.51

$-34.57$

-31.21
-31.15

$-27.02$

21.18
$-\quad 20.94$

$-18.82$ 


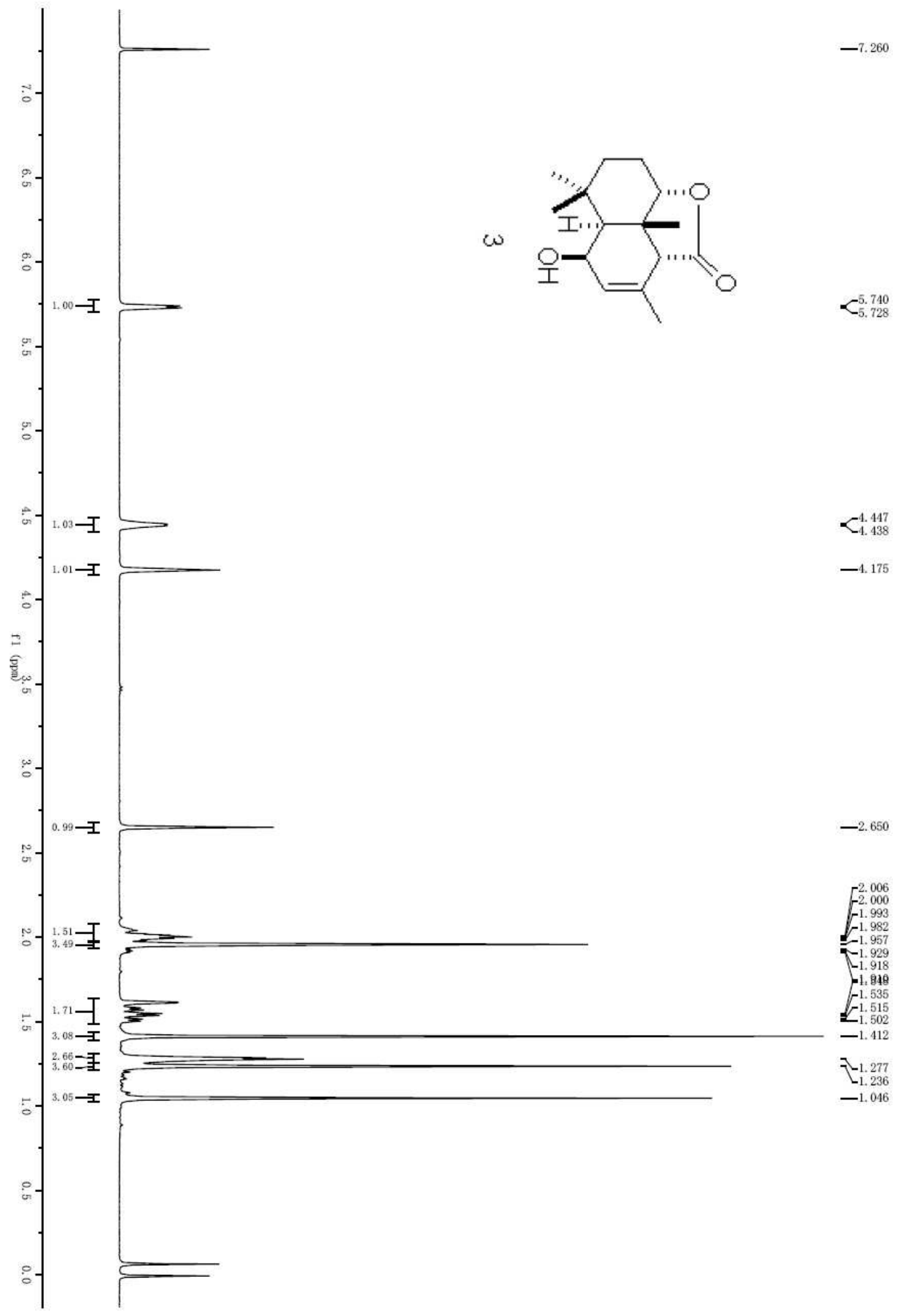




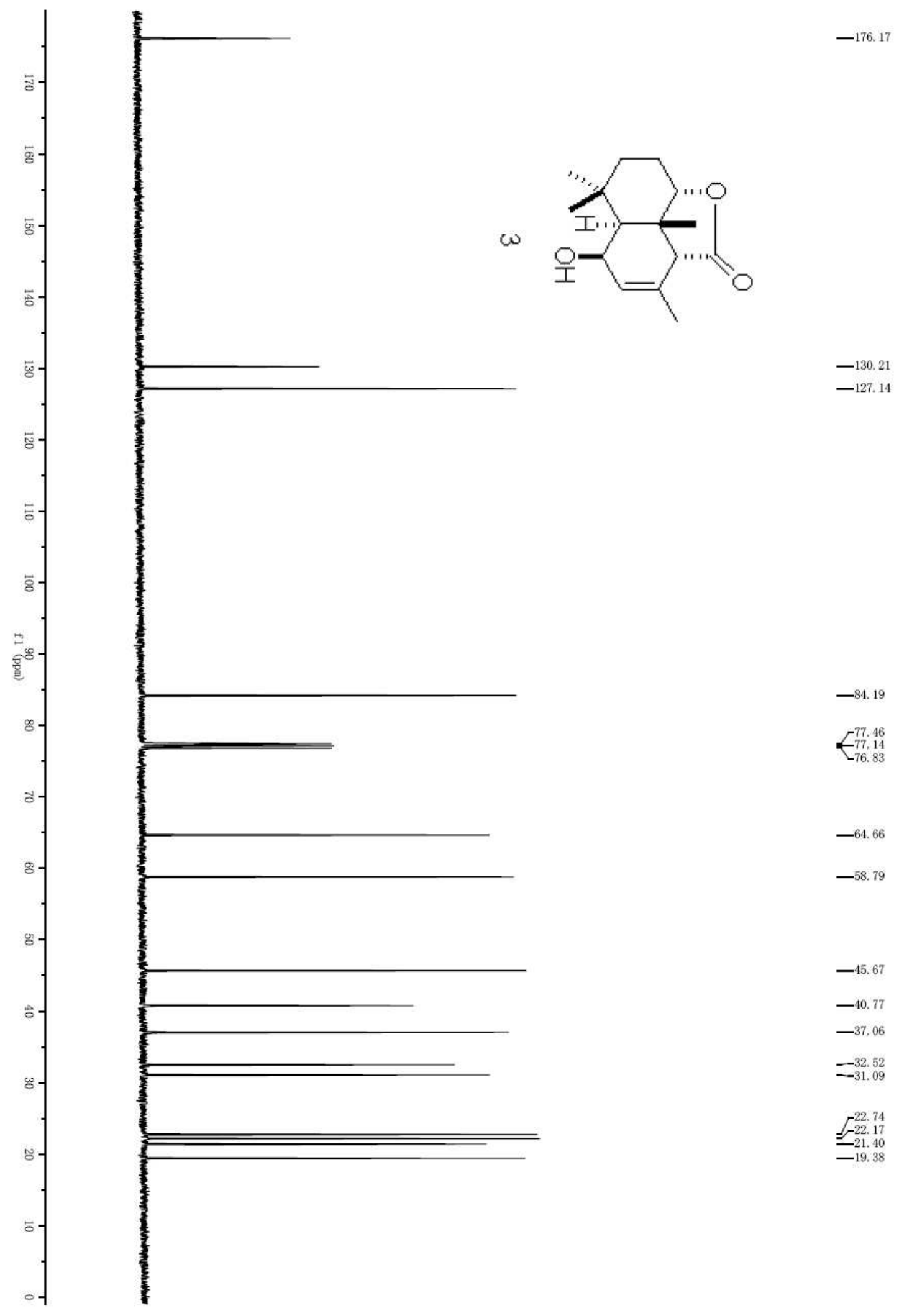




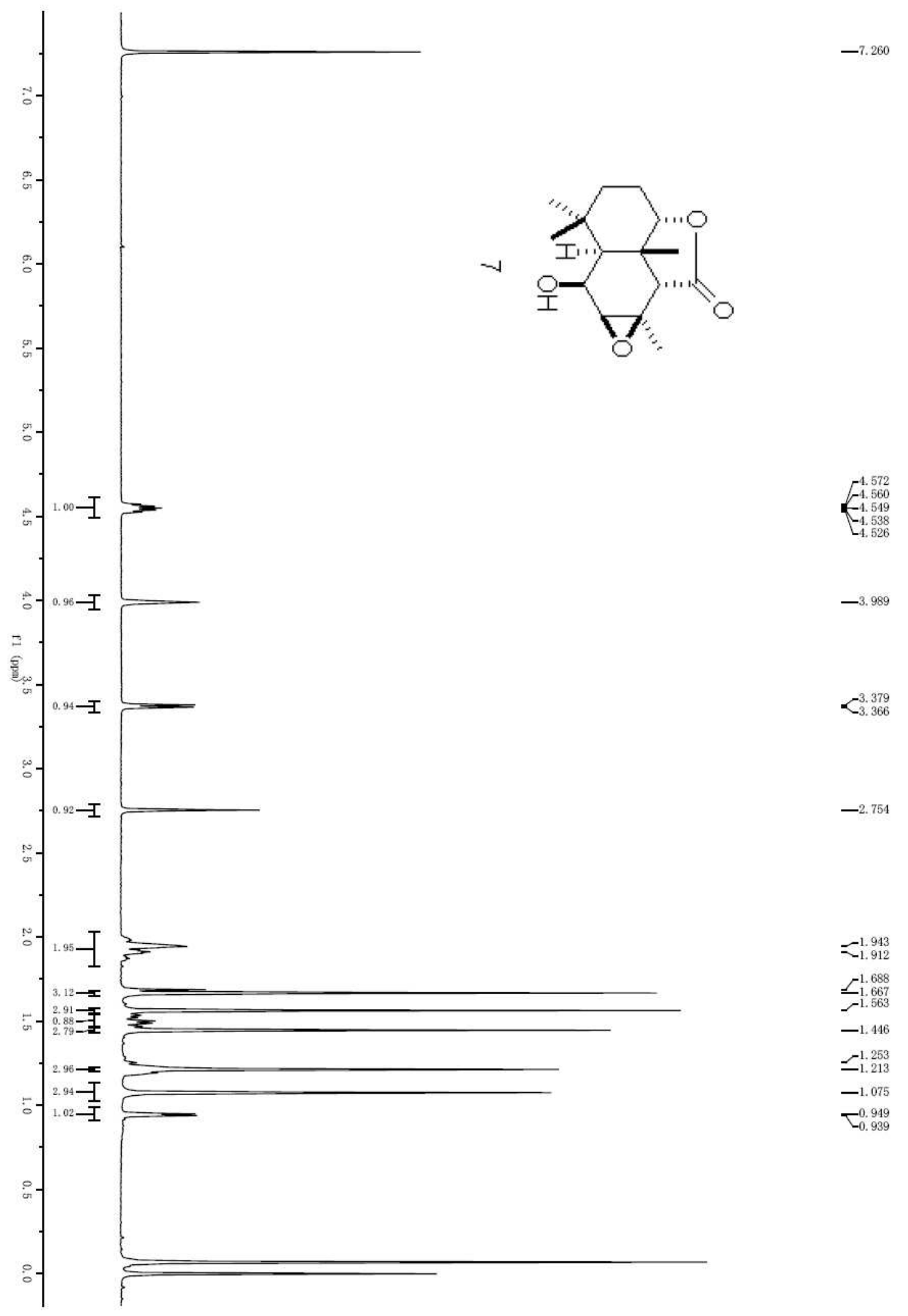




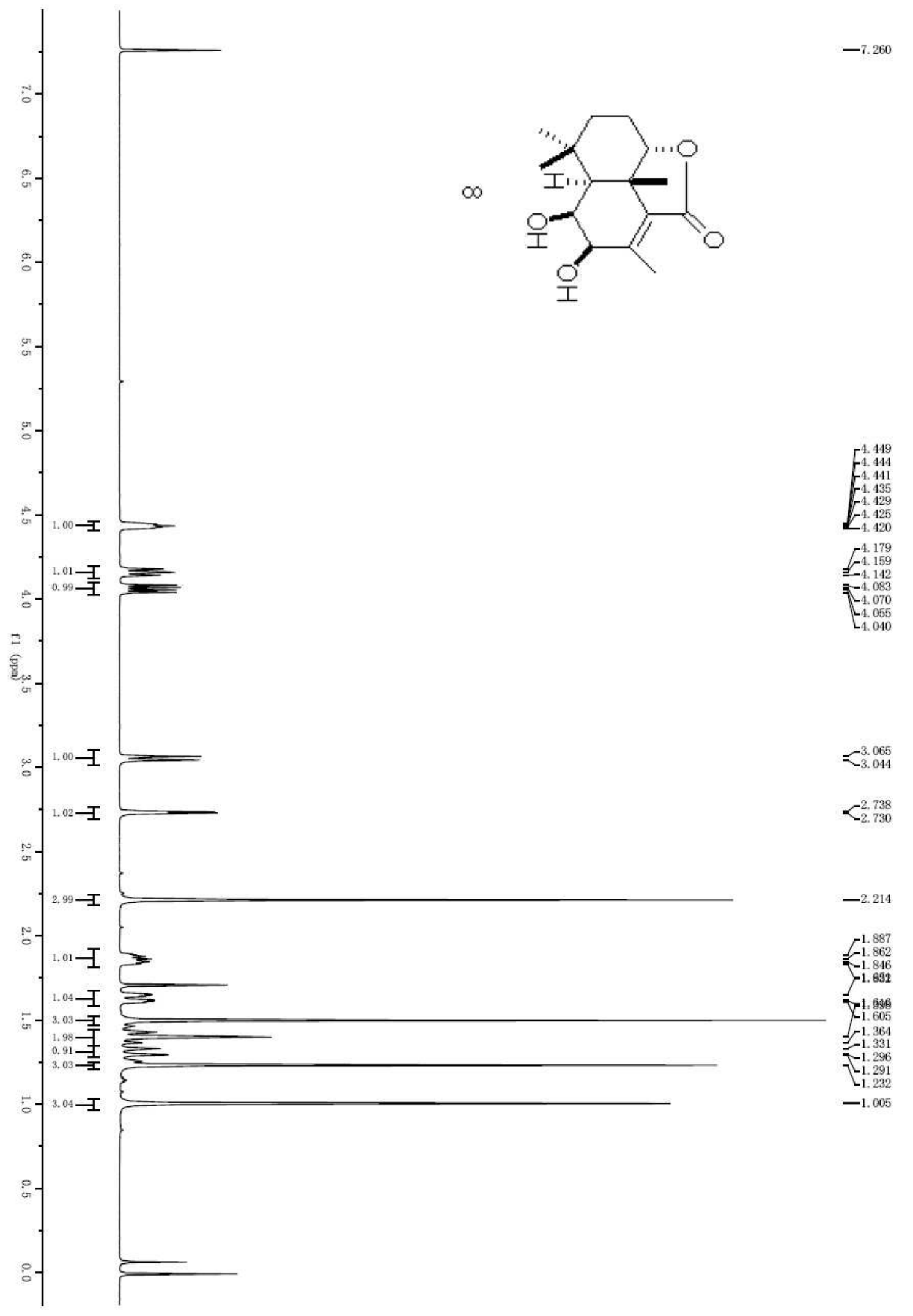




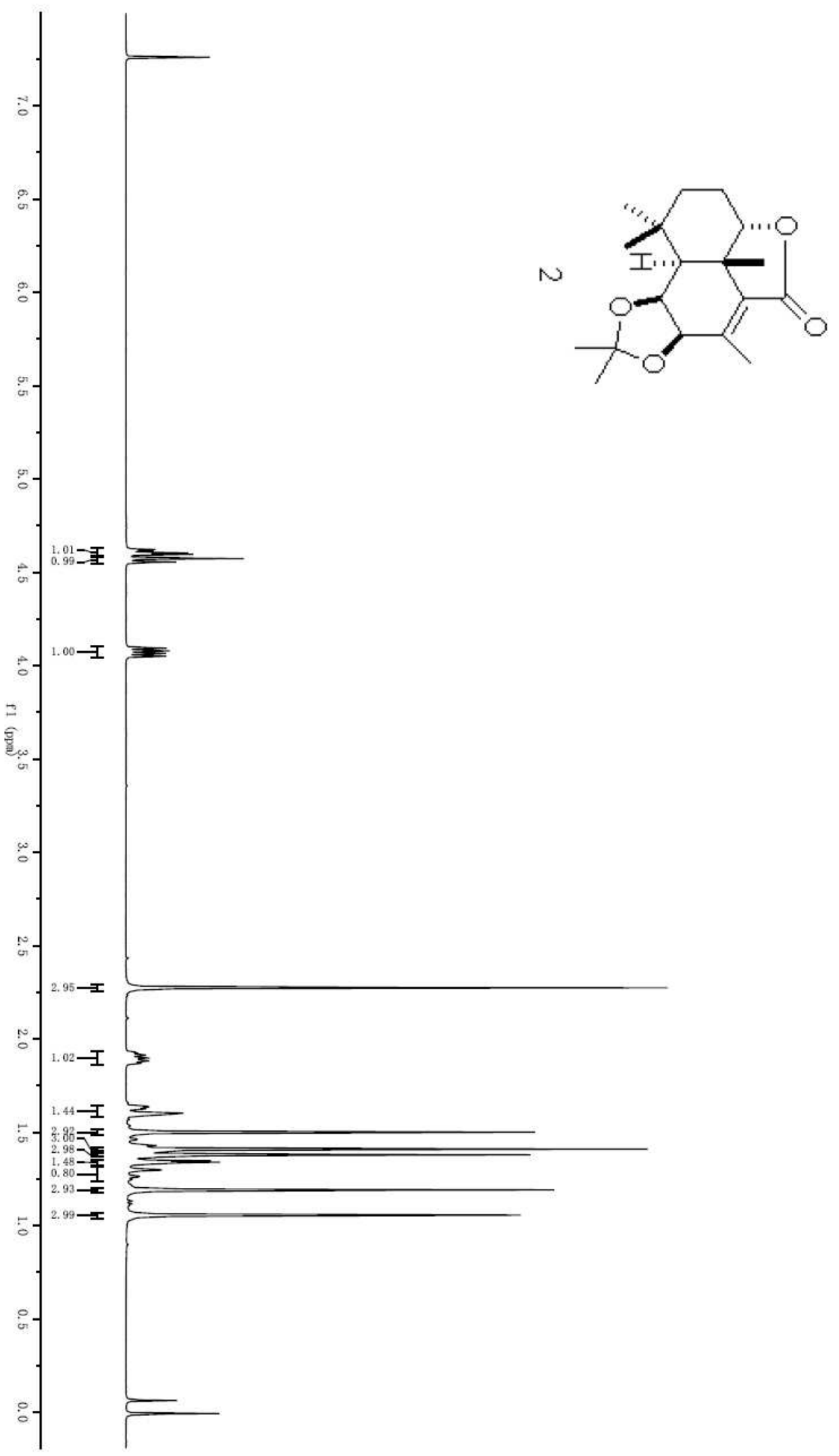

$-7.260$

4.623
-4.615
-4.604

-4.597

$-4.574$

$C^{4.0994}$

$\underbrace{-4.060}_{-.051}$

$-2.274$

${ }_{-1.998}^{1.914}$

- -1.892

$-1.882$

-1.822
-1.602

$-1.459$

$\mathcal{Z}_{-1.340}^{1.410}$

$-1.191$

$-1.056$ 

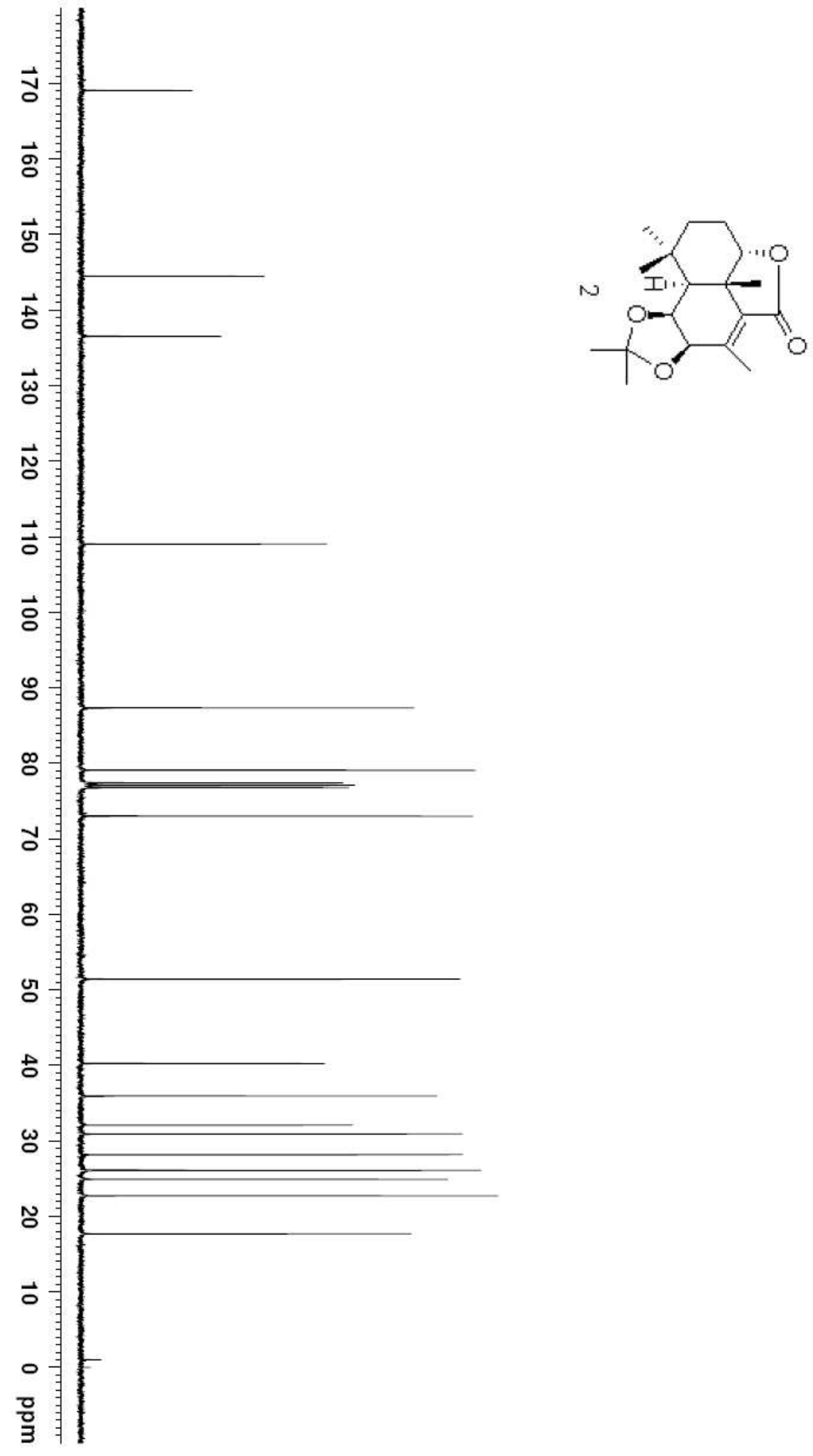

$-144.47$

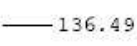

$-109.05$

$-87.33$

79.10
-77.40

77.40
-77.09

77.09
-76.77

$-73.00$

$-51.43$

40.26

35.94

$-32.07$

$-30.91$

$-28.17$

26.13
-26.10

24.93
-22.73

$-17.69$

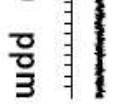

